\title{
A CLASS OF INTEGER VALUED ENTIRE FUNCTIONS $\left({ }^{1}\right)$
}

\author{
BY \\ AFTON H. CAYFORD
}

Introduction. The entire functions of a complex variable which are the subject of this paper might be considered as a class of integral valued entire functions. The functions we study are those which, together with all of their derivatives, take on Gaussian integer values on a finite point set in the plane. An example of this type of function is $f(z)=\exp z(z-1)$ which, along with its derivatives, is integral at $z=0$ and $z=1$.

Work has been done to establish lower bounds for the order and type for such transcendental entire functions by Straus [1] and in this paper we further clarify the relationship between the order of the function and the corresponding point set by using methods similar to those used in the study of integral valued entire functions. We consider the scale of possible values for $\rho$ and observe that it contains certain critical values. Functions with order less than one are polynomials while there may exist transcendental functions of order greater than or equal to one. We define $\rho_{c}$ to be a number, greater than or equal to one, with the property that there are no functions with order between one and $\rho_{c}$. Lower bounds for the value of $\rho_{c}$ are obtained for certain types of point set and our conjecture is that $\rho_{c}$ is equal to $s$, the number of points in the set, in general $\left({ }^{2}\right)$. The number $s$ is also critical and for a fixed value of $s$ the number of possible functions with order less than $s$ is countable while the number with any given order greater than or equal to $s$ is uncountable. We show that if $t$ such functions, all of order no greater than $\rho$, take on, together with all of their derivatives, Gaussian integer values on a set of $s$ points, then, if $\rho$ is less than $s-\left[(s-1) \sqrt{ } s /(s+t)^{1 / 2}\right]$ there exist $t$ linear differential operators with integral coefficients, $L_{1}, \ldots, L_{t}$, such that $L_{1} f_{1}+\cdots+L_{t} f_{t}=0$. We also find that if $t$ such functions have order at most $\rho$ where $\rho$ is less than $s(1-1 / t)$ then they are algebraically dependent.

We investigate the characteristics of certain differential rings that these functions might belong to. In particular we look at a differential ring of entire functions with

Presented to the Society, June 16, 1961 under the title Entire functions which, together with all of their derivatives, assume Gaussian integer values on a finite point set; received by the editors December 4, 1966 and, in revised form, June 11, 1968.

(') Much of the contents of this paper formed the author's 1961 U.C.L.A. Ph.D. Thesis which was directed to Dr. E. G. Straus. The author is heavily indebted to both Dr. Straus and the referee for assistance with this manuscript.

$\left.{ }^{2}\right)$ This conjecture has now been proved. 
the properties that each element satisfies a linear differential equation with constant coefficients and the degree of transcendentality is one. We show that it is either a ring of polynomials or that each element of this ring is a linear combination of exponentials of the form $\exp (r \beta z)$ where $r$ is rational and $\beta$ is a constant, fixed for the ring. Also if the ring contains a transcendental integral valued function of our type we see that $\beta$ is either a rational integer or the square root of a rational integer.

I. Order. Let $f(z)$ be entire and suppose that there exists a positive number $A$ such that $f(z)=O\left(\exp r^{A}\right)$ as $|z|=r \rightarrow \infty$. Let $\rho$ be the greatest lower bound of the set of such numbers $A$. Then $\rho$ is the order of $f(z)$. Thus, $\rho$ is the least number for which $f(z)=O\left(\exp r^{\rho+\mathscr{E}}\right)$ for all $\mathscr{E}>0$ as $r \rightarrow \infty$, or, equivalently,

$$
\rho=\lim _{r \rightarrow \infty} \sup \frac{\log \log M(r)}{\log r}
$$

where

$$
M(r)=\max _{|z|=r}|f(z)| .
$$

In a sense, this subject begins with a theorem by Pólya [2] which was proved by Hardy [3]. It is

THEOREM 1.1. If $f(z)$ is an entire function satisfying the condition

$$
\lim _{r \rightarrow \infty} \sup \frac{\log M(r)}{r}<\log 2
$$

and if $f(0), f(1), \ldots$ are integers, then $f(z)$ is a polynomial.

In [4] T. Schneider has proved a comprehensive theorem on integral valued functions which states that under certain complicated conditions a set of $n$ meromorphic functions is algebraically dependent. Although this theorem is not used directly, the method of proof underlies that one used in proving the major theorems of this paper.

Several theorems in a paper by Straus [1] are of particular interest to us and in essence form a background for this present work. The first of these, due to Pólya and Kakeya [5], [6] is as follows:

LEMMA 1.2. If $f(z)$ is a transcendental entire function and $f^{(n)}(0)$ is integral for $n=0,1, \ldots$ then $f(z)$ is at least of order 1 type 1.

The proof of the portion of this theorem involving order will be reproduced from [1] here to illustrate the general way in which the condition of integral value is used throughout this paper.

Proof. Let $f(z)=\sum_{n=1}^{\infty} c_{n} z^{n}$. Then $f^{(n)}(0)=n ! c_{n}$ and hence $c_{n}=a_{n} / n$ ! where $a_{n}$ is integral. Thus we have either $c_{n}=0$ or $\left|c_{n}\right| \geqq 1 / n !$. Since $f(z)$ is not a polynomial, 
there exists an infinite sequence of $n$ for which the latter inequality holds. The order $\rho$ is given by

$$
\rho=\lim _{n \rightarrow \infty} \sup \frac{n \log n}{-\log \left|c_{n}\right|} \geqq \lim _{n \rightarrow \infty} \sup \frac{n \log n}{\log n !}=1
$$

The second theorem, which is given as Theorem 1 of [1] is

THEOREM 1.3. If $f(z)$ is a transcendental entire function and $f^{(n)}(z)$ is integral for $z=0, \ldots, s-1 ; n=0,1, \ldots$ then $f(z)$ is at least of order $s$ type $[(s-1) !]^{-2}$.

It is this estimate of the order of $f(z)$ that gives rise to the first question, which is: what effect would the removal of the constraint that the set of points at which the function is integral valued be integers have on the conclusion? In order to attempt to answer this question and to generally clarify the relationship between the order and the number $s$, we will need the following theorem.

THEOREM 1.4. Let $f(z)$ be an entire function and let $\left\{z_{i}\right\} ; i=1, \ldots, s$ be a finite set of points. Then $f(z)$ can be represented by a series of the form

$$
f(z)=\sum_{m=0: m=k s+n}^{\infty} a_{m}\left(z-z_{1}\right)^{k+1} \cdots\left(z-z_{n}\right)^{k+1}\left(z-z_{n+1}\right)^{k} \cdots\left(z-z_{s}\right)^{k}
$$

where $1 \leqq n \leqq s, k=0,1, \ldots$

Proof. For the sake of convenience we write $z_{m}=z_{n}$ for $m \equiv n(\bmod s), 1 \leqq n \leqq s$, so that (1.1) becomes

$$
f(z)=\sum_{m=0}^{\infty} a_{m} \prod_{\mu=1}^{m}\left(z-z_{\mu}\right)
$$

Now we have

$$
\frac{1}{t-z}=\sum_{\mu=1}^{m} \prod_{v=1}^{\mu} \frac{z-z_{v}}{t-z_{v}} \frac{1}{t-z_{\mu+1}}+\prod_{\mu=1}^{m+1} \frac{z-z_{\mu}}{t-z_{\mu}} \cdot \frac{1}{t-z} .
$$

This is proved by induction. For $m=0$ we have

$$
\frac{1}{t-z}=\frac{z-z_{1}}{\left(t-z_{1}\right)(t-z)}+\frac{1}{t-z_{1}}
$$

If (1.2) holds for $m$ then it holds for $m+1$ if we replace the factor $1 /(t-z)$ in the last term by the equivalent expression

$$
\frac{1}{t-z_{m+1}}+\frac{z-z_{m+1}}{\left(t-z_{m+1}\right)(t-z)}
$$


Now let $C$ be the contour $|t|=r$ with $r>\left|z_{i}\right|, i=1, \ldots, s$. By Cauchy's theorem we have for $|z|<r$

$$
\begin{aligned}
f(z)=\frac{1}{2 \pi i} \int_{C} \frac{f(t)}{t-z} d t= & \sum_{\mu=0}^{m} \prod_{v=1}^{\mu}\left(z-z_{v}\right) \frac{1}{2 \pi i} \int_{C} f(t) \prod_{v=1}^{\mu+1}\left(t-z_{v}\right)^{-1} d t \\
& +\prod_{\mu=1}^{m+1}\left(z-z_{\mu}\right) \frac{1}{2 \pi i} \int_{C} f(t) \prod_{\mu=1}^{m+1}\left(t-z_{\mu}\right)^{-1} \frac{d t}{t-z} \\
= & \sum_{\mu=0}^{m} a_{\mu} \prod_{v=1}^{\mu}\left(z-z_{v}\right)+R_{m}
\end{aligned}
$$

where

$$
a_{u}=\frac{1}{2 \pi i} \int_{C} f(t) \prod_{v=1}^{\mu+1}\left(t-z_{v}\right)^{-1} d t
$$

and

$$
R_{m}=\frac{1}{2 \pi i} \int_{C} f(t) \prod_{\mu=1}^{m+1} \frac{z-z_{\mu}}{t-z_{\mu}} \frac{d t}{t-z}
$$

We now estimate $R_{m}$, choosing $r$ so large that $r>5 \max \left\{|z|,\left|z_{1}\right|, \ldots,\left|z_{s}\right|\right\}$. Then for $|t|=r$ we have $|t-z|>r / 2$ and $\left|t-z_{i}\right|>2\left|z-z_{i}\right|, i=1, \ldots, s$. Now, if $M(r)$ $=\max _{|t|=r}|f(t)|$ we have

$$
\begin{aligned}
\left|R_{m}\right| & \leqq \frac{1}{2 \pi} \int|f(t)| \prod_{\mu=1}^{m+1}\left|\frac{z-z_{\mu}}{t-z_{\mu}}\right|\left|\frac{1}{t-z}\right||d t| \\
& \leqq 2^{-(m+1)} M(r) \frac{2 \pi r}{2 \pi} \cdot \frac{2}{r}=M(r) 2^{-n_{\iota}} \rightarrow 0 \text { as } m \rightarrow \infty
\end{aligned}
$$

Therefore

$$
\begin{aligned}
f(z)=a_{0}+a_{1}\left(z-z_{1}\right)+\cdots+ & a_{k s+n}\left(z-z_{1}\right)^{k+1} \cdots \\
& \left(z-z_{n}\right)^{k+1}\left(z-z_{n+1}\right)^{k} \cdots\left(z-z_{s}\right)^{k}+\cdots .
\end{aligned}
$$

We have seen by means of Lemma 1.2 that if the order $\rho$ of one of our functions is less than one then the function is a polynomial. We will now use Theorem 1.4 to establish another critical point in the range of values of $\rho$. Suppose we have the family $F_{s \rho}$ of functions of order at most $\rho$ so that at some $s$ points they and all of their derivatives are Gaussian integer valued. We can show that if $\rho<s$ then $F_{s \rho}$ is countable and if $\rho \geqq s$ then $F_{s \rho}$ is uncountable. This means that we cannot hope to characterize functions of order greater than or equal to $s$ by means of any condition of a countable type. For example, we cannot say that such a function must satisfy some algebraic differential equation with integer coefficients.

In order to prove this, let $f(z)$ be entire and together with all of its derivatives take on Gaussian integer values on the point set $\left\{z_{i}\right\} ; i=1, \ldots, s$. Using the series representation (1.1) of Theorem 1.4, we get 


$$
\begin{aligned}
f^{(m)}\left(z_{n}\right)= & P_{m s+n-1}+a_{m s+n-1} m !\left(z_{n}-z_{1}\right)^{m+1} \ldots \\
& \left(z_{n}-z_{n-1}\right)^{m+1}\left(z_{n}-z_{n+1}\right)^{m} \cdots\left(z_{n}-z_{s}\right)^{m} \\
= & P_{m s+n-1}+T_{m s+n-1},
\end{aligned}
$$

where $P_{m s+n-1}$ includes only terms $a_{i}$ with $i<m s+n-1$. We will show that when $f(z)$ is of order $\rho$ and $\rho<s$ we have $\left|T_{m s+n-1}\right|<1 / 2$ if $m$ is sufficiently large and therefore

$$
\left|f^{(m)}\left(z_{n}\right)-P_{m s+n-1}\right|<1 / 2 .
$$

Now since $f^{(m)}\left(z_{n}\right)$ is a Gaussian integer and the value of $P_{m s+n-1}$ is determined by the choice of the $m s+n-1$ coefficients preceding $a_{m s+n-1}$ we have only one possible choice for the value of $f^{(m)}\left(z_{n}\right)$ once the values $a_{\mu}, \mu<m s+n-1$ have been chosen. This in turn determines the value of $a_{m s+n-1}$.

Thus we see that the function $f(z)$ is completely determined after a finite number of the coefficients in its series expansion have been chosen. This also means that a function $f(z)$ is completely determined after the values have been chosen for a finite number of its derivatives, and since these are all integers we can have at most countably many different functions.

We will also show that for every $\rho$ with $\rho \geqq s$ we can pick families of sequences of coefficients $a_{i}$ so that we have at least two choices for each $a_{i}$ and so that the $f(z)$ given by (1.1) are of order $\rho$ and have integer valued derivatives of all orders at $z_{1}, \ldots, z_{s}$.

For the proof of these inequalities we will need the following lemma.

LEMMA 1.5. A necessary and sufficient condition that a function represented by the series (1.1) of Theorem 1.4 be an entire function of finite order $\rho$ is that

$$
\lim _{n \rightarrow \infty} \inf \frac{\log \left(1 /\left|a_{n}\right|\right)}{n \log n}=\frac{1}{\rho}
$$

Proof. The proof is essentially the same as that for power series given in [7]. Let

$$
\lim _{n \rightarrow \infty} \inf \frac{-\left(\log \left|a_{n}\right|\right)}{n \log n}=u \text {. }
$$

Where $u$ is 0 , positive, or infinite. Then, for every positive $\mathscr{E}$,

$$
-\log \left|a_{n}\right|>(u-\mathscr{E}) n \log n \quad(n>p)
$$

or

$$
\left|a_{n}\right|<n^{-n(u-8)}
$$

If $u>0$, the series converges for all values of $z$ so that $f(z)$ is an entire function. Also, if $u$ is finite, let $|z|=r$ then for large $r>1$ we have $\left|z-z_{i}\right|<2 r$ and therefore

$$
\left|a_{s k+n}\left(z-z_{1}\right)^{k+1} \cdots\left(z-z_{n}\right)^{k+1}\left(z-z_{n+1}\right)^{k} \cdots\left(z-z_{s}\right)^{k}\right| \leqq\left|a_{k s+n}\right|(2 r)^{s k+n} .
$$


This gives $|f(z)|<A(2 r)^{p}+\sum_{m=p+1}^{\infty}(2 r)^{m} m^{-m(u-8)}$. Let $S_{1}$ denote the part of this latter sum for which $m \leqq(4 r)^{1 /(u-8)}$ and $S_{2}$ the remainder. Then in $S_{1}$

$$
(2 r)^{m} \leqq \exp \left\{(4 r)^{1 /(u-8)} \log 2 r\right\}
$$

so that

$$
S_{1}<\exp \left\{(4 r)^{1 /(u-\varnothing)} \log (2 r)\right\} \sum m^{-m(u-8)}<K \exp \left\{(4 r)^{1 /(u-8)} \log (2 r)\right\} .
$$

In $S_{2}$

$$
2 r m^{-(u-8)}<2 r\left((4 r)^{1 /(u-8)}\right)^{-u+8}=1 / 2
$$

so that $S_{2}<\sum(1 / 2)^{m}<1$.

Therefore

$$
|f(z)|<K \exp \left\{(4 r)^{1 /(u-8)} \log (2 r)\right\}
$$

which means $\rho \leqq 1 /(u-\mathscr{E})$. Letting $\mathscr{E} \rightarrow 0$ gives $\rho \leqq 1 / u$. If $u=\infty$ the argument with an arbitrarily large number instead of $u$ shows that $\rho=0$.

On the other hand, given $\mathscr{E}>0$, there is a sequence of values of $m$ for which

$$
-\log \left|a_{m}\right|<(u+\mathscr{E}) m \log m \text { or }\left|a_{m}\right|>m^{-m(u+\mathscr{E})} .
$$

By considering the definition of $a_{m}$ in Theorem 1.4, we see that if $M(r)$ is the maximum of $|f(z)|$ on a circle of radius $r$ we have

$$
M(r) \geqq\left|a_{m}\right| r^{m} 2^{-m-1}
$$

and if we take $r=2(2 m)^{u+8}$ we have

$$
\begin{aligned}
M(r) & \geqq \frac{\left|a_{m}\right|}{2}\left(\frac{2(2 m)^{u+\varnothing}}{2}\right)^{m}>\frac{m^{-m(u+\varnothing)}}{2}(2 m)^{m(u+\varnothing)} \\
& =2^{m(u+\varnothing)-1}=\exp \{(m(u+\mathscr{E})-1) \log 2\} \\
& =\frac{1}{2} \exp \left\{(u+\mathscr{E}) 2^{-(1+1 /(u+\varnothing))}(\log 2) r^{1 /(u+\varnothing)}\right\}>\exp \left\{A r^{1 /(u+\varnothing)}\right\}
\end{aligned}
$$

Since this holds for a sequence of values of $r$ tending to infinity, we have $\rho \geqq 1 /(u+\mathscr{E})$ and letting $\mathscr{E} \rightarrow 0$ gives $\rho \geqq 1 / u$. If $u=0$, the argument shows that $f(z)$ is of infinite order.

We now have

$$
\begin{aligned}
\left|T_{m s+n-1}\right| & =\left|a_{m s+n-1} m !\left(z_{n}-z_{1}\right)^{m+1} \cdots\left(z_{n}-z_{n-1}\right)^{m+1}\left(z_{n}-z_{n+1}\right)^{m} \cdots\left(z_{n}-z_{s}\right)^{m}\right| \\
& <(m s+n-1)^{-(m s+n-1)(1 / \rho-8)} m^{m} h^{m s+n-1} \\
& <(m s+n-1)^{m(1-s / \rho+s \&)}(m s+n-1)^{-(n-1)(1 / \rho-8)} h^{m s+n-1}
\end{aligned}
$$

for $m$ sufficiently large and $h=\max \left|z_{i}-z_{j}\right| ; i, j=1, \ldots, s ; \mathscr{E}>0$. Now if $\rho<s$ the exponent of $m s+n-1$ is negative for $\mathscr{E}$ sufficiently small and therefore $\left|T_{m s+n-1}\right|$ $<1 / 2$, if $m$ is sufficiently large. 
On the other hand, we can choose the coefficients $a_{m}$ so that the

$$
\lim _{m \rightarrow \infty} \sup \frac{m \log m}{-\log \left|a_{m}\right|}=\rho \geqq s
$$

and at the same time $\left|T_{m}\right|>1$ for all sufficiently large $m$. We can then modify $T_{m}$ by an amount $T_{m}^{1}$ whose absolute value is less than one to make the corresponding value of the derivative, which equals $P_{m}+T_{m}$, a Gaussian integer. There are at least two such choices for $T_{m}^{1}$ for each $m=0,1,2, \ldots$ thus constructing $2^{\mathrm{x}_{0}}$ different entire functions. Since $\left|T_{m}^{1}\right|<1$ the corresponding change $a_{m}^{1}$ in $a_{m}$ satisfies

$$
\lim _{n \rightarrow \infty} \sup \frac{m \log m}{-\log \left|a_{m}^{1}\right|} \leqq s
$$

so that the modified functions have order less than or equal to $\rho$, where inequality holds for at most a countable set of the $2^{\mathrm{x}_{0}}$ functions constructed.

In this paper we will not consider values of $\rho$ which are greater than $s$ but will establish some conditions on functions with $1 \leqq \rho<s$. For example, we have the following

THEOREM 1.6. If $f(z)$ is an entire function of order $\rho$ with the property that at the points $z=0, \alpha$ all of the values of $f$ and its derivatives are Gaussian integers and if $1 \leqq \rho<2-(2 / 3)^{1 / 2}$ then $f(z)$ satisfies a linear differential equation with Gaussian integral coefficients.

This theorem is a special case of Theorem 2.1 which will be proved later.

This example shows that there is a gap in the possible values for the order running from 1 up to a number we call $\rho_{c}$. In Theorem 1.6 we obtain a lower bound of $2-(2 / 3)^{1 / 2}$ for $\rho_{c}$ but our conjecture (see footnote 2 ) is that $\rho_{c}=s$ in general as it does in the case of Theorem 1.3. We are investigating the characteristics of functions with order in this range by considering the problem from the point of view of the relationships among several such functions. These results are given in the following section.

\section{Differential dependence.}

THEOREM 2.1. If $f_{i}(z), i=1, \ldots, t$ are entire functions of orders $\leqq \rho$ with the property that at the $s$ points $\left\{z_{r}\right\}, r=1, \ldots, s$ all of the values $f_{i}^{(n)}\left(z_{r}\right), i=1, \ldots, t$; $r=1, \ldots, s ; n=0,1, \ldots$ are Gaussian integers and

$$
\rho<s-(s-1)(s /(s+t))^{1 / 2},
$$

then, if $M$ is sufficiently large there exist Gaussian integers $C_{i j}$, not all zero, such that

$$
\Phi(z)=\sum_{i=1}^{t} \sum_{j=0}^{M} C_{i}, f_{i}^{(j)}(z)
$$

is identically zero. 
Proof. The proof will be in two parts. We let $M=[q m]$ where $q$ is a constant to be determined later and we shall first show (Lemma 2.3) that if we choose an $m$ sufficiently large there exist Gaussian integers $C_{i j}$ for $j=0, \ldots$, [qm] for which the function

$$
\Phi(z)=\sum_{i=1}^{t} \sum_{j=0}^{[q m]} C_{i j} f_{i}^{(j)}(z)
$$

has the property that $\Phi^{(k)}\left(z_{r}\right)=0 ; r=1, \ldots, s ; k=0, \ldots, m-1$; and obtain an estimate of the size of the integers $C_{i j}$.

Secondly, we shall show (Lemma 2.4) that if $m$ is sufficiently large we have $\Phi^{(n)}\left(z_{r}\right)=0, r=1, \ldots, s ; n \geqq m$ and hence $\Phi(z)$ is identically zero.

LEMMA 2.2. If $f(z)$ is an entire function of order $\leqq \rho$ where $\rho \geqq 1$ then for any point $z_{0}$ we have

$$
\left|f^{(n)}\left(z_{0}\right)\right|<n^{n\left(1-1 /\left(\rho+\varepsilon_{1}\right)\right)}
$$

for all $\varepsilon_{1}>0$ and all $n>n_{0}\left(\varepsilon_{1}, z_{0}\right)$.

Proof. This is a special case of Lemma 1.5 , since $f^{(n)}\left(z_{0}\right) / n$ ! is the $n$th coefficient of the Taylor series at $z_{0}$. Therefore

$$
\left|f^{(n)}\left(z_{0}\right) / n !\right|<n^{-n /\left(\rho+\varepsilon_{1}\right)}
$$

for large $n$ and

$$
\left|f^{(n)}\left(z_{0}\right)\right|<n ! n^{-n /\left(\rho+\varepsilon_{1}\right)}<n^{n\left(1-1 /\left(\rho+\varepsilon_{1}\right)\right)}
$$

for large $n$.

LEMMA 2.3. If $f_{1}(z), \ldots, f_{t}(z)$ are entire functions of order $\leqq \rho$ and with the property that at the $s$ points $z_{1}, \ldots, z_{s}$ we have $f_{i}^{(n)}\left(z_{r}\right)$ Gaussian integers for all $n=0,1,2, \ldots ; i=1, \ldots, t ; r=1, \ldots, s$ then there exist Gaussian integers $C_{i j}$ not all zero such that

$$
\Phi(z)=\sum_{i=1}^{t} \sum_{j=0}^{[q m]} C_{i j} f_{i}^{(j)}(z)
$$

has the property $\Phi^{(k)}\left(z_{r}\right)=0$ for $k=0,1, \ldots, m-1 ; r=1, \ldots, s$; and such that

$$
\left|C_{i j}\right|<m^{\lambda\left(\left(\left(t q^{2}+s\right) / 2(t q-s)\right) m-j\right)}
$$

where $\lambda=1-1 /(\rho+\mathscr{E})$ and $q$ is a positive constant to be determined later.

Proof. Consider the sm linear expressions

$$
y_{r k}=\sum_{i=1}^{t} \sum_{j=0}^{[q m]} x_{i j} f_{i}^{(j+k)}\left(z_{r}\right), \quad k=0, \ldots, m-1 ; r=1, \ldots, s,
$$

where $x_{i j}$ is a Gaussian integer $a_{i j}+b_{i j} i$ with $0 \leqq a_{i j}, b_{i j} \leqq h_{j}-1$. This gives a total number of right-hand sides in (2.1) of

$$
\left(\prod_{j=0}^{[q m]} h_{j}^{2}\right)^{t}
$$


Now by Lemma 2.2, we have

$$
\left|y_{r k}\right| \leqq \sum_{i=1}^{t} \sum_{j=0}^{[q m]}\left|x_{i j}\right|\left|f_{i}^{(j+k)}\left(z_{r}\right)\right| \leqq t c_{1} \sum_{j=0}^{[q m]} h_{j}(j+k)^{(j+k)(1-1 /(\rho+\xi))}
$$

where $c_{1}$ is a constant independent of $j$ and $k$, so that the number of different $s m$ tuples on the left side of $(2.1)$ is no greater than

$$
\left(t^{s m} c^{s m}\left(\prod_{k=0}^{m-1} \sum_{j=0}^{[a m]} h_{j}(j+k)^{(j+k) \lambda}\right)^{s}\right)^{2}
$$

where $\lambda=(1-1 /(\rho+\mathscr{E}))$ and $c=2 c_{1}$. Now let $h_{j}=H \cdot m^{-\lambda j}$, which is possible provided

$$
H \cdot m^{-\lambda q m}>1 \text {. }
$$

If we estimate the size of $\prod_{j=0}^{[q m]} h_{j}^{2 t}$ we get

$$
\prod_{j=0}^{[q m]} h_{j}^{2 t}=\prod_{j=0}^{[q m]}\left(H^{t} m^{-\lambda t j}\right)^{2}
$$

We also find that (2.2) can be estimated by

$$
\begin{aligned}
\left(t^{s m} c^{s m} \prod_{k=0}^{m-1}\left(\sum_{j=0}^{[q m]} H m^{-\lambda j}(j+k)^{(j+k) \lambda}\right)^{s}\right)^{2} & =\left(t^{s m} c^{s m} \prod_{k=0}^{m-1}\left(H \sum_{j=0}^{[q m]}\left(\frac{j+k}{m}\right)^{\lambda j}(j+k)^{\lambda k}\right)^{s}\right)^{2} \\
& =\left(t^{s m} c^{s m} \prod_{k=0}^{m-1}\left(H \sum_{j=0}^{[q m]} m^{o(m)} m^{\lambda k}\right)^{s}\right)^{2} \\
& =\left(H^{s m} m^{\lambda s m^{2} / 2+o\left(m^{2}\right)}\right)^{2} .
\end{aligned}
$$

Therefore the number of distinct $y$ vectors will be less than the number of distinct $x$ vectors if

$$
H^{s m} m^{\lambda s m^{2} / 2+o\left(m^{2}\right)} \leqq H^{q m t+o(m)} m^{-\lambda t q^{2} m^{2} / 2}
$$

or

$$
H^{q m t-s m+o(m)} \geqq m^{m^{2}\left(\left(t q^{2}+s\right) / 2\right) \lambda+o\left(m^{2}\right)}
$$

or

$$
H=m^{\lambda m\left(\left(t q^{2}+s\right) / 2(t q-s)\right)+o(m)} \quad \text { if } q t>s
$$

where, because of the $\mathscr{E}$ in $\lambda$ we can ignore the $o(m)$ term for sufficiently large $m$.

Therefore, there must be two distinct $x$ vectors which give the same $y$ vector and if we choose our coefficients $C_{i j}$ to be the components of the difference vector of these two vectors we will have a function $\Phi(z)$ with the required properties, and with

$$
\left|C_{i j}\right| \leqq m^{\lambda\left(\left(\left(t q^{2}+s\right) / 2(t q-s)\right) m-j\right)} \text {. }
$$

The condition (2.3) will be satisfied if $\left(t q^{2}+s\right) / 2(t q-s)-q \geqq 0$ that is,

$$
q \leqq\left(s+\left(s^{2}+t s\right)^{1 / 2}\right) / t
$$


We note that the upper bound on the $C_{i j}$ is then an increasing function of $m$.

LeMMA 2.4. If $\rho<s-(s-1)(s /(s+t))^{1 / 2}$ and $m$ is sufficiently large then the function $\Phi(z)$ defined in Lemma 2.3 vanishes identically.

Proof. We shall prove that, for $m$ sufficiently large, we have $\Phi^{(m)}\left(z_{r}\right)=0$, $r=1, \ldots, s$, and hence by induction that $\Phi^{(p)}\left(z_{r}\right)=0$. For $p=0,1, \ldots ; r=1, \ldots, s$ so that $\Phi \equiv 0$. We consider the function $\Phi(z) \prod_{r=1}^{s}\left(z-z_{r}\right)^{-m}$ and note that since $\Phi(z)$ has a zero of order at least $m$ at each point $z_{r} ; r=1, \ldots, s$ this function is entire. Therefore we can write

$$
\begin{aligned}
\Phi^{(m)}\left(z_{r}\right) & =\left.\frac{d^{m}}{d z^{m}}\left(\frac{\Phi(z)}{\prod_{p \neq r}\left(z-z_{p}\right)^{m}}\right)\right|_{z=z_{r}} \cdot \prod_{p \neq r}\left(z_{r}-z_{p}\right)^{m} \\
& =\frac{m ! \prod_{p \neq r}\left(z_{r}-z_{p}\right)^{m}}{2 \pi i} \cdot \int_{|z|=R} \frac{\Phi(z) d z}{\prod_{p \neq r}\left(z-z_{p}\right)^{m}\left(z-z_{r}\right)^{m+1}} \\
& =\frac{m ! \prod_{p \neq r}\left(z_{r}-z_{p}\right)^{m}}{2 \pi i} \sum_{i=1}^{t} \sum_{j=0}^{[a m]} C_{i j} \int_{|z|=R} \frac{f_{i}^{(j)}(z) d z}{\prod_{p=1}^{s}\left(z-z_{p}\right)^{m}\left(z-z_{r}\right)}
\end{aligned}
$$

where $R>a=\max \left\{\left|z_{p}\right|, p=1, \ldots, s\right\}$.

If for each $i$ and $j$ we now perform an integration by parts $j$ times we can write this in the form

$$
=\frac{m ! \prod_{p \neq r}\left(z_{r}-z_{p}\right)^{m}}{2 \pi i} \sum_{i=1}^{t} \sum_{j=0}^{[q m]}(-1)^{j} C_{i j} \cdot \int_{|z|=R} f_{i}(z) \frac{d^{j}}{d z^{j}}\left(\prod_{p=1}^{s}\left(z-z_{p}\right)^{-m}\left(z-z_{r}\right)^{-1}\right) d z .
$$

We must now obtain an upper bound for the size of $\left|\Phi^{(m)}\left(z_{r}\right)\right|$ and to do this we note that by hypothesis we have

$$
\left|f_{i}(z)\right|<e^{|z| \rho+8}, \quad i=1, \ldots, t
$$

for $|z|=R$ and $R \geqq R_{0}$, and by Lemma 2.3 we have

$$
\left|C_{i j}\right|<m^{\lambda\left(\left(\left(t q^{2}+s\right) / 2(t q-s)\right) m-j\right)} .
$$

In estimating

$$
\left|\frac{d^{j}}{d z^{j}} \prod_{p=1}^{s}\left(z-z_{p}\right)^{-m}\left(z-z_{r}\right)^{-1}\right|
$$

we consider the function as a product of terms of the form $\left(z-z_{i}\right)^{-1}$, so that using the product rule of differentiation we get $(s m+1) \cdots(s m+j)$ terms each of which has absolute value no greater than $(R-a)^{-(s m+j+1)}$ so that

$$
\left|\frac{d^{j}}{d z^{j}} \prod_{p=1}^{s}\left(z-z_{p}\right)^{-m}\left(z-z_{r}\right)^{-1}\right| \leqq \frac{(s m+j) !}{(s m) !}(R-a)^{-(s m+j+1)} .
$$


We now use these to estimate $\left|\Phi^{(m)}\left(z_{r}\right)\right|$ and get

$$
\left|\Phi^{(m)}\left(z_{r}\right)\right|<\frac{m^{m} c^{m}}{2 \pi} \sum_{i=1}^{t} \sum_{j=0}^{[q m]} m^{\lambda\left(\left(\left(t q^{2}+s\right) / 2(t q-s)\right) m-j\right)} 2 \pi R e^{R \rho+8} \frac{(s m+j)^{j}}{(R-a)^{s m+j+1}} .
$$

Now choose $m$ large enough so that $R_{0}^{\rho+8}<m$ and set $R=m^{1 /(\rho+8)}$. We get

$$
\left|\Phi^{(m)}\left(z_{r}\right)\right|<m^{o(m)} m^{m\left(1+\lambda\left(t q^{2}+s\right) / 2(t q-s)\right)} \sum_{i=1}^{t} \sum_{j=0}^{[q m]} m^{-j \lambda} m^{1 /(\rho+\mathscr{E})} e^{m} \frac{(s m+j)^{j}}{\left(m^{1 /(\rho+8)}-a\right)^{s m+j+1}} .
$$

Since $a<\frac{1}{2} m^{1 /(\rho+8)}$ for large enough $m$ and $j \leqq q m$ we can write this as

$$
\begin{aligned}
& <m^{o(m)} m^{m\left(1+\lambda\left(t q^{2}+s\right) / 2(t q-s)\right)} t \sum_{j=0}^{[q m]} m^{j(-1+1 /(\rho+\mathscr{E})+1-1 /(\rho+\mathscr{8}))} \\
& \cdot m^{-s m /(\rho+8)-1 /(\rho+8)+1 /(\rho+\varnothing)} e^{m} 2^{s m+j+1}(s+q)^{j} \\
& =m^{o(m)} m^{m\left(1+\lambda\left(t q^{2}+s\right) / 2(t q-s)-s /(\rho+8)\right)} .
\end{aligned}
$$

Substituting $(1-1 /(\rho+\mathscr{E}))$ for $\lambda$ we find that the exponent on $m$ is negative whenever

$$
\rho+\mathscr{E}<\frac{\left(\left(t q^{2}+s\right) / 2(t q-s)\right)+s}{\left(\left(t q^{2}+s\right) / 2(t q-s)\right)+1}=1+\frac{s-1}{\left(\left(t q^{2}+s\right) / 2(t q-s)\right)+1} .
$$

If we now consider the expression on the right as a function of $q$ we find that it has a maximum value for $q=\left(s+\left(s^{2}+t s\right)^{1 / 2}\right) / t$.

Therefore, whenever

$$
\rho<\left(s-(s-1)(s /(s+t))^{1 / 2}\right)=\rho_{t}
$$

the exponent on $m$ will be negative for some $\mathscr{E}>0$ and for $m$ sufficiently large we have $\left|\Phi^{(m)}\left(z_{r}\right)\right|<1$, and since this is a nonnegative integer, it must be zero. The point $z_{r}$ was chosen arbitrarily from among the $s$ points $z_{p}(p=1, \ldots, s)$ and so we have $\Phi^{(m)}\left(z_{p}\right)=0 ; p=1, \ldots, s$.

In the proof of Lemma 2.4 we have shown that if the function $\Phi(z)$ of Lemma 2.3 has a zero of order $m$ at each of the points $z_{p}, p=1, \ldots, s$ then it also has a zero of order $m+1$ at each of them. We can now repeat this proof of Lemma 2.4 replacing $m$ by $m+1$ and obtain a zero of order $m+2$. Therefore, by induction, $\Phi(z)$ has a zero of every order at each of the points $z_{p} ; p=1, \ldots, s$ and therefore is identically zero.

As $t \rightarrow \infty$ we see that $\rho_{t} \rightarrow s$. Thus, if we define $R_{\rho}$ as the ring of all entire functions of order $\leqq \rho$ which have integral derivatives at the points $z_{1}, \ldots, z_{s}$ then for $\rho<s$ this ring is a finite dimensional module over the linear differential operators with Gaussian integral coefficients.

\section{Algebraic dependence.}

THEOREM 3.1. If $f_{1}, \ldots, f_{t}$ are entire functions with the property that the numbers $f_{i}^{(n)}\left(z_{r}\right)$ are Gaussian integers for $i=1, \ldots, t ; r=1, \ldots, s ; n=0,1, \ldots$ and $\left|f_{i}(z)\right|$ $<\exp \left(|z|^{\rho+\mathscr{E}}\right)(i=1, \ldots, t)$ for $|z|>r_{0}(\mathscr{E})$ and $\rho<s(1-1 / t)$ then the functions are algebraically dependent over the integers. 
Proof. The proof will be by means of an induction. We shall first show that if $M$ is sufficiently large there exist Gaussian integers $C_{u_{1} \ldots u_{t}}$, not all zero, for which the function

$$
\Phi(z)=\sum_{u_{1}=0}^{M-1} \cdots \sum_{u_{t}=0}^{M-1} C_{u_{1} \ldots u_{t}} f_{1}^{u_{1}} \cdots f_{t}^{u_{t}}
$$

has the property that $\Phi^{(n)}\left(z_{r}\right)=0$ for $r=1, \ldots, s ; n=0, \ldots, m-1$; and obtain an estimate of the size of the $C_{u_{1} \ldots u_{t}}$.

Secondly, we shall show that if $m$ is chosen sufficiently large we have $\Phi^{(n)}\left(z_{r}\right)=0$, $r=1, \ldots, s$; for all $n \geqq m$ and hence $\Phi(z)$ is identically zero.

LEMMA 3.2. If $f_{1}(z), \ldots, f_{t}(z)$ are entire functions of order $\leqq \rho$ where $\rho \geqq 1$ then at any $z_{0}$ we have

$$
\left.\left|\frac{d^{n}}{d z^{n}} f_{1}^{u}(z) \cdots f_{t}^{u}(z)\right|\right|_{z=z_{0}}<n^{n\left(1-1 /\left(\rho+\mathscr{E}_{1}\right)\right)} b^{n /\left(\rho+\&_{1}\right)}
$$

where $b=u_{1}+\cdots+u_{t}$ for all $\mathscr{E}_{1}>0$ and all $n>n_{0}\left(\mathscr{E}_{1}, z_{0}\right)$.

Proof. By Cauchy's integral formula we have

$$
\frac{d^{n}}{d z^{n}} f_{1}^{u_{1}} \cdots f_{t}^{u_{t}}\left(z_{0}\right)=\frac{n !}{2 \pi i} \int_{\left|z-z_{0}\right|=R} \frac{f_{1}^{u_{1}} \cdots f_{t}^{u_{t}}}{\left(z-z_{0}\right)^{n+1}} d z
$$

so that

$$
\left|\frac{d^{n}}{d z^{n}} f_{1}^{u_{1} \cdots} \cdot f_{t^{u}}^{u_{t}}\left(z_{0}\right)\right|<\frac{n^{n} 2 \pi R \exp \left[b\left(R+\left|z_{0}\right|\right)^{\rho+\&}\right]}{2 \pi R^{n+1}} .
$$

If we choose $R=(n / b)^{1 /(\rho+8)}$ we get

$$
\begin{aligned}
\left|\frac{d^{n}}{d z^{n}} f_{1}^{u_{1}} \cdots f_{t}^{u}\left(z_{0}\right)\right| & <n^{n(1-1 /(\rho+\varnothing))} b^{(n /(\rho+\varnothing))} e^{n\left(1+\left|z_{0}\right| / R\right)^{\rho+\varnothing}} \\
& =n^{n(1-1 /(\rho+\varnothing))+o(n)} b^{n /(\rho+\varnothing)}<n^{n\left(1-1 /\left(\rho+\S_{1}\right)\right.} b^{n /\left(\rho+\varnothing_{1}\right)}
\end{aligned}
$$

if $n$ is sufficiently large.

LEMMA 3.3. If $f_{1}, \ldots, f_{t}$ are entire functions with the property that the numbers $f_{i}^{(n)}\left(z_{r}\right)$ are Gaussian integers for $i=1, \ldots, t ; r=1, \ldots, s ; n=0, \ldots$ and such that for any $\varepsilon>0$ there exist $r(\varepsilon)$ such that $\left|f_{i}(z)\right|<\exp \left(|z|^{\rho+\varepsilon}\right)$ for $|z|>r(\varepsilon)$ and $i=1, \ldots, t$ then there exist Gaussian integers $C_{u_{1} \ldots u_{t}}$, not all zero, such that the function

$$
\Phi(z)=\sum_{u_{1}=0}^{M-1} \cdots \sum_{u_{t}=0}^{M-1} C_{u_{1} \ldots u_{t}} f_{1}^{u_{1}} \cdots f_{t}^{u_{t}}(z)
$$

has the property $\Phi^{(n)}\left(z_{r}\right)=0 ; r=1, \ldots, s ; n=0, \ldots, m-1$ and

$$
\left|C_{u_{1} \ldots u_{t}}\right|<m^{(m s / 2(q-s))(1-(1-1 / t) /(\rho+\varepsilon))}
$$

where $M=\left[(q m)^{1 / t}\right]+1$ with $q$ a positive constant to be determined later. 
Proof. For ease of computation we shall assume some ordering of the subscripts so that we can write the expression for $\Phi$ as

$$
\Phi(z)=\sum_{u} C_{u} w_{u}(z)
$$

where $u$ takes on $M^{t}$ different values. We now consider the set of $s m$ homogeneous linear equations in $M^{t}$ unknowns $\Phi^{(n)}\left(z_{r}\right)=0$. If we now let $M=\left[(q m)^{1 / t}\right]+1$ we can consider the $s m$ tuples, not necessarily all different, defined by the equations $y_{r n}=\sum_{u=1}^{M^{t}} x_{u} w_{u, n}\left(z_{r}\right)$ where the $x_{u}$ are Gaussian integers $A_{u}+B_{u} i$ and $w_{u, n}$ is the $n$th derivative of $w_{u}$. Suppose $0 \leqq A_{u}, B_{u} \leqq H-1$. Then the total number of different $M^{t}$ tuples $\left\{x_{u}\right\} ; u=1, \ldots, M^{t}$ is

$$
\prod_{u=1}^{M^{t}} H^{2} \geqq H^{2 q m}
$$

We must now estimate the size of an individual value $y_{r n}$. In order to do this we must estimate the size of the term $w_{u, n}\left(z_{r}\right)$. This term is of the form

$$
\left.\frac{d^{n}}{d z^{n}} f_{1}^{u_{1}} \cdots f_{t}^{u} t(z)\right|_{z=z_{r}}
$$

We can now use Lemma 3.2 and obtain

$$
\left|w_{u, n}\left(z_{r}\right)\right|<C n^{n(1-1 /(\rho+\varepsilon))} b^{n /(\rho+\varepsilon)}, \quad r=1, \ldots, s,
$$

where $C$ is independent of $m$ and is necessary for those terms where $n<n_{0}$ of Lemma 3.2.

We have that

$$
\left|y_{r n}\right| \leqq \sum_{u=1}^{M^{t}} H C n^{\lambda n} b(u)^{n /(\rho+\varepsilon)} \leqq H C n^{\lambda n}(t M)^{n /(\rho+\varepsilon)} M^{t}
$$

where $\lambda=1-1 /(\rho+\varepsilon)$, so that the number of different sm tuples $\left\{y_{r n}\right\}$ is no greater than

$$
\begin{aligned}
\left(\left(H 2 C M^{t}\right)^{s m} \prod_{n=1}^{m-1} n^{\lambda n s}(t M)^{s n /(\rho+\varepsilon)}\right)^{2} & <\left(\left(H 2 C M^{t}\right)^{s m}(t M)^{\left(m^{2} / 2\right)(s /(\rho+\varepsilon))} m^{\left(\lambda s m^{2} / 2\right)+o\left(m^{2}\right)}\right)^{2} \\
& \leqq\left(\left(H 2 C 2^{t} q m\right)^{s m}\left(2 t(q m)^{1 / t}\right)^{m^{2} s / 2(\rho+\varepsilon)} m^{\left(\lambda s m^{2} / 2\right)+o\left(m^{2}\right)}\right)^{2} \\
& =\left(H^{s m} m^{\left(\lambda s m^{2} / 2\right)+\left(m^{2} s / 2 t(\rho+\varepsilon)\right)+o\left(m^{2}\right)}\right)^{2} .
\end{aligned}
$$

The number of distinct $y$ vectors $\left\{y_{r n}\right\}$ will be less than the number of distinct $x$ vectors $\left\{x_{u}\right\}$ if

$$
H^{q m} \geqq H^{s m} m^{\left(\lambda s m^{2} / 2\right)+\left(m^{2} s / 2 t(\rho+\varepsilon)\right)+o\left(m^{2}\right)}
$$

which can be satisfied by

$$
H=m^{(m s / 2(q-s))(\lambda+1 / t(\rho+\varepsilon))+o(m)}
$$

if $q>s$ and where because of the $\varepsilon$ we can ignore the $o(m)$ term for sufficiently large $m$. Therefore, for this $H$, there must be two distinct $x$ vectors which give the same $y$ 
vector and if we choose the coefficients $C_{u}$ to be the components of the difference vector of these two $x$ vectors we will have a function $\Phi(z)$ with the required properties and with

$$
\left|C_{u}\right| \leqq m^{(m s / 2(q-s))(1-(1-1 / t) /(\rho+\varepsilon))}
$$

LEMMA 3.4. If $\rho<s(1-1 / t)$ and $m$ is sufficiently large then the function $\Phi(z)$ defined in Lemma 3.3 vanishes identically.

Proof. We shall prove that for $m$ sufficiently large we have $\Phi^{(m)}\left(z_{r}\right)=0$, $r=1, \ldots, s$, and hence by induction that $\Phi^{(p)}\left(z_{r}\right)=0$ for $p=0,1, \ldots ; r=1, \ldots, s$ so that $\Phi \equiv 0$. We consider the function $\Phi(z) \prod_{r=1}^{s}\left(z-z_{r}\right)^{-m}$ and since this function has a zero of order at least $m$ at each point $z_{r}, r=1, \ldots, s$, it is entire and we can write

$$
\begin{aligned}
\Phi^{(m)}\left(z_{r}\right) & =\left.\frac{d^{m}}{d z^{m}}\left(\frac{\Phi(z)}{\prod_{p \neq r}\left(z-z_{p}\right)^{m}}\right)\right|_{z=z_{r}} \prod_{p \neq r}\left(z_{r}-z_{p}\right)^{m} \\
& =\frac{m ! \prod_{p \neq r}\left(z_{r}-z_{p}\right)^{m}}{2 \pi i} \int_{|z|=R} \frac{\Phi(z) d z}{\prod_{p \neq r}\left(z-z_{p}\right)^{m}\left(z-z_{r}\right)^{m+1}} \\
& =\frac{m ! \prod_{p \neq r}\left(z_{r}-z_{p}\right)^{m}}{2 \pi i} \sum_{u_{1}=0}^{M-1} \cdots \sum_{u_{t}=0}^{M-1} C_{u_{1} \ldots u_{t}} \int_{|z|=R} \frac{f_{1}^{u_{1}}(z) \cdots f_{t}^{u}(z) d z}{\prod_{p=1}^{s}\left(z-z_{p}\right)^{m}\left(z-z_{r}\right)} .
\end{aligned}
$$

We must now obtain an upper bound for this expression and in order to do this we note that by hypothesis we have

$$
\left|f_{i}(z)\right|<\exp \left(|z|^{o+\mathscr{E}}\right) ; \quad i=1, \ldots, t
$$

for large $|z|$ and by Lemma 3.3 we have

$$
\left|C_{u_{1} \ldots u_{t}}\right| \leqq m^{(m s / 2(q-s))(1-(1-1 / t) /(\rho+8))} \quad(q>s) .
$$

Now let $a$ be chosen so that $a \geqq\left|z_{p}\right|$ for $p=1, \ldots, s$ and we then have $\left|z-z_{p}\right| \geqq R-a$ for $p=1, \ldots, s$. Using these estimates we now have

$$
\begin{aligned}
&\left|\Phi^{(m)}\left(z_{r}\right)\right|>\frac{m ! \prod_{p \neq r}\left|z_{r}-z_{p}\right|^{m}}{2 \pi} \sum_{u_{1}=0}^{M-1} \cdots \sum_{u_{t}=0}^{M-1} m^{(m s / 2(q-s))(1-(1-1 / t) /(\rho+8))} \\
& . \frac{2 \pi R C \exp \left[t(M-1) R^{\rho+8}\right]}{(R-a)^{s m+1}} .
\end{aligned}
$$

Now let $R=\left(m^{1-1 / t}\right)^{1 /(\rho+8)}$ and we get

$$
\left|\Phi^{(m)}\left(z_{r}\right)\right|<m^{m} K^{m} q m m^{(m s / 2(q-s))(1-(1-1 / t) /(\rho+\varnothing))} \frac{C \exp \left[t q^{1 / t} m\right]}{\left(m^{1-1 / t}\right)^{m s /(\rho+8)}}
$$


since $a<\frac{1}{2} R$ for $m$ sufficiently large. We can now write this as

$$
\begin{aligned}
\left|\Phi^{(m)}\left(z_{r}\right)\right| & <m^{o(m)} m^{m}\left(1+\frac{s}{2(q-s)}\left(1-\frac{1}{\rho+8}\left(1-\frac{1}{t}\right)\right)-\frac{s}{\rho+8}\left(1-\frac{1}{t}\right)\right) \\
& =m^{o(m)} m^{m}\left(\frac{2 q-s}{2(q-s)}-\frac{1}{\rho+\varnothing}\left(1-\frac{1}{t}\right)\left(\frac{s}{2(q-s)}+s\right)\right) \\
& =m^{o(m)} m^{m}\left(\frac{2 q-s}{2(q-s)}-\frac{s}{\rho+8}\left(1-\frac{1}{t}\right)\left(\frac{1+2 q-2 s}{2(q-s)}\right)\right) \\
& =m^{o(m)} m \frac{m}{2(q-s)}\left(2 q-s-\frac{s}{\rho+8}\left(1-\frac{1}{t}\right)(1+2 q-2 s)\right) .
\end{aligned}
$$

The coefficient of the exponent $m$ becomes negative if

$$
2 q-s-\frac{s}{\rho+\mathscr{E}}\left(1-\frac{1}{t}\right)(1+2 q-2 s)<0
$$

or

$$
\rho+\mathscr{E}<\frac{s(1-1 / t)(1+2 q-2 s)}{2 q-s}=s\left(1-\frac{1}{t}\right)\left(1-\frac{s-1}{2 q-s}\right)
$$

and this can be made as close to $s(1-1 / t)$ as we choose if $q$ is chosen sufficiently large.

Therefore, if for a given $s$ and $t$ we have $\rho<s(1-1 / t)$, we can choose a value for $q$ so that for sufficiently large $m$ the corresponding function $\Phi(z)$ satisfies inequality (3.1) above with the exponent of $m$ negative for some $\mathscr{E}>0$. We therefore have $\left|\Phi^{(m)}\left(z_{r}\right)\right|<1$ for $m$ sufficiently large and since this is a nonnegative integer it must be zero. The point $z_{r}$ was chosen arbitrarily from among the $s$ points $z_{p}, p=1, \ldots, s$ and so we have $\Phi^{(m)}\left(z_{p}\right)=0, p=1, \ldots, s$.

In the proof of Lemma 3.4 we have shown that if the function $\Phi(z)$ of Lemma 3.3 has a zero of order $m$ at each of the points $z_{p} ; p=1, \ldots, s$ then it also has a zero of order $m+1$ at each of them. We can now repeat this proof of Lemma 3.4 replacing $m$ by $m+1$ and obtain a zero of order $m+2$. Therefore, by induction, $\Phi(z)$ has a zero of every order at each of the points $z_{p} ; p=1, \ldots, s$ and therefore is identically zero.

IV. It may be possible to use some combination of these theorems to raise the lower bound for $\rho_{c}$. We can let the functions $f_{i}(z)$ of Theorem 3.1 be a function $f(z)$ and some of its derivatives to obtain an algebraic differential equation which must be satisfied by a single function. In this case the order may be low but the degree high. We can obtain an algebraic differential equation of low degree which must be satisfied by $f(z)$ if $\rho<s$ if we let

$$
f_{1}(z)=f(z), \quad f_{2}(z)=(f(z))^{2}, \ldots, f_{t}(z)=\left(f^{(t-1)}(z)\right)^{2}
$$

in Theorem 2.1 but the order may be high. Since functions $f(z)$ of this type retain their properties under differentiation, sum, and product an interesting question which arises is: What sort of differential ring might such a function belong to? 
It will certainly belong to some members of the following family $\mathscr{F}$ of differential rings.

Let $\mathscr{F}$ be a family of rings $A$, closed under differentiation and with the following three properties. If $f \in A$ then $f$ is an entire function. For a given ring $A$ there exist constants $p$ and $t$ such that if $f_{1}, \ldots, f_{t}$ are elements of $A$ then there exist $t$ linear differential operators with Gaussian integer coefficients $L_{1}, \ldots, L_{t}$, not all trivial, such that $L_{1} f_{1}+\cdots+L_{t} f_{t}=0$. Every set of $p$ elements of $A$ is algebraically dependent. We can examine the structure of certain members of this family $\mathscr{F}$. more closely by means of the following theorem for the case $p=2, t=1$.

THEOREM 4.1. Let $A$ be a differential ring of entire functions which is an algebra over a ring $R$ of complex numbers with the property that every function in $A$ satisfies a linear differential equation with constant coefficients and every two functions are algebraically dependent over $A$. Then either $A$ is a ring of polynomials or there exists a nonzero constant $\beta$ so that every $f \in A$ has the form

$$
f(z)=\sum_{i=1}^{n} c_{i} e^{r_{i} \beta z}
$$

where the $c_{i}$ are constants and the $r_{i}$ are rational.

If $R$ is the ring of integers and there exists an $f \in A$ which together with all its derivatives is integral at some point $z_{0}$, then $f(z)$ is a polynomial with rational coefficients either in $z-z_{0}$ or in $e^{z-z_{0}}$ and $e^{-\left(z-z_{0}\right)}$ or in $\cosh \left(\sqrt{ } d\left(z-z_{0}\right)\right)$ and $(1 / \sqrt{ } d) \sinh \left(\sqrt{ } d\left(z-z_{0}\right)\right)$ where $d$ is a (squarefree) integer.

Proof. To prove the first part of the theorem it suffices to consider the case in which $A$ is an algebra over the complex numbers, since both the linear differential equations and the algebraic dependence properties are preserved if we extend $A$ to an algebra over $C$ and the conclusion for the extended algebra implies the conclusion for $A$.

Now every $f(z) \in A$ has the form

$$
f(z)=\sum_{i=1}^{n} p_{i}(z) e^{v_{i}(z)}
$$

where the $p_{i}$ are polynomials of degree $m_{i}$. Applying the linear operator

$$
L=\prod_{i \neq k}\left(D-v_{i}\right)^{m_{i}+1} \cdot\left(D-v_{k}\right)^{m_{k}}
$$

we get $L f=c e^{v_{k} z}$ where $c$ is a nonzero constant. Hence $e^{v_{i} z} \in A ; i=1, \ldots, n$. Now two functions $e^{a z}, e^{\beta z}$ are algebraically dependent if and only if $a, \beta$ are nontrivial rationally dependent. Thus there exists a $\beta$ such that all $\nu_{i}$ have the form $r_{i} \beta, r_{i}$ rational.

If $m_{k} \geqq 1$ for some $k$, then applying

$$
L_{1}=\prod_{i \neq k}\left(D-v_{i}\right)^{m_{i}+1}\left(D-v_{k}\right)^{m_{k}-1}
$$


we get

$$
L_{1} f=\left(c_{1} z+c_{0}\right) e^{v_{k} z}, \quad c_{1} \neq 0 .
$$

Thus $z$ is in the quotient field of $A$ and all elements of $A$ are algebraically dependent on $z$; that is, $A$ is a ring of polynomials. This completes the proof of the first part of the theorem.

To prove the second part of the theorem we assume without loss of generality that $z_{0}=0$. If $f(z)$ is a polynomial in $z$ then the coefficients $f^{(n)}(0) / n$ ! are rational by hypothesis.

Now assume that $f(z)=\sum_{i=1}^{n} c_{i} e^{r_{i} \beta z}$. Since $f$ has integral valued derivatives at 0 there exists a nontrivial linear differential operator $L$ with integral coefficients so that

$$
L f(0)=L f^{\prime}(0)=\cdots=L f^{(n)}(0)=0
$$

and therefore $L f \equiv 0$. This shows that $\beta$ is algebraic. If the $r_{i} \beta$ are not algebraic conjugates, then there exist linear differential operators with integral coefficients which annihilate some of the $e^{r_{i} \beta z}$ but not others. Applying such an operator, we get another nonzero function of the form $f(z)=\sum_{j=1}^{m} c_{j}^{\prime} e^{r_{j} \beta z}$ where $m<n$. We therefore may restrict attention to the case in which all the $r_{i} \beta$ are algebraic conjugates. Now if $r_{i} \beta$ is a conjugate of $r_{j} \beta$, that is, $\beta$ is a conjugate of $r \beta$ where $r=r_{i} / r_{j}$, then it is also conjugate of $r^{n} \beta$ with $n=0, \pm 1, \ldots$. This is possible only if $r= \pm 1$. If $r=1$, then there exists an $f(z)=c e^{\beta z}, c \neq 0$, so that $f^{(n)}(0)=c \beta^{n}$ is integral for all $n=0,1, \ldots$ This is clearly possible only if $c$ and $\beta$ are integral. Thus, in this case $A$ is a ring of polynomials with rational coefficients in $e^{z}$ and $e^{-z}$.

If $r=-1$, then $\beta$ and $-\beta$ are conjugates; in other words, $\beta=\sqrt{ } d$ where $d$ is a nonsquare rational. Then $f(z)$ has the form

$$
f(z)=c_{1} e^{\beta z}+c_{2} e^{-\beta z}, \quad\left(c_{1}, c_{2}\right) \neq(0,0),
$$

$f^{(n)}(0)=c_{1} \beta^{n}+c_{2}(-\beta)^{n}$ is an integer for $n=0,1,2, \ldots$ This is clearly possible only if $\beta$ is an algebraic integer; that is, $d$ is a nonsquare integer, and $c_{1}, c_{2}$ are conjugate elements of $Q(\sqrt{ } d)$. In other words

$$
\begin{aligned}
f(z) & =a(\exp (\sqrt{ } d z)+\exp (-\sqrt{ } d z))+b \sqrt{ } d(\exp (\sqrt{ } d z)-\exp (-\sqrt{ } d z)) \\
& =2 a \cosh (\sqrt{ } d z)+2 b \sqrt{ } d \sinh (\sqrt{ } d z)
\end{aligned}
$$

where $a, b$ are rationals. This completes the proof.

According to Theorem 1.6 all entire functions of order $\rho<2-\sqrt{ } 6 / 3$ which, together with all their derivatives, are integral valued at 0 and $\alpha, \alpha \neq 0$, satisfy linear differential equations with integral coefficients. Now, if there existed two algebraically independent entire functions of this kind, then as was shown in the proof of Theorem 4.1 there would exist two entire functions $f(z), g(z)$ with algebraic derivatives of all orders at 0 and $\alpha$, one a nonconstant polynomial with algebraic coefficients in $e^{\beta z}$ and $e^{-\beta z}, \beta$ algebraic, $\beta \neq 0$; and the other either a nonconstant polynomial with algebraic coefficients in $z$, or a nonconstant polynomial with 
algebraic coefficients in $e^{\gamma z}$ and $e^{-\gamma z}$ for some algebraic $\gamma$ with $\gamma / \beta$ irrational. Since no two of the quantities $\alpha, e^{\alpha \beta}, e^{\alpha \gamma}$ can be simultaneously algebraic for $\alpha \neq 0$ this leads to a contradiction. Thus the hypotheses of Theorem 4.1 hold and combining this with Theorem 1.6, we have characterized all points $\alpha$ so that there exist nonconstant entire functions $f(z)$ of order $\rho<2-\sqrt{ } 6 / 3$ which, together with all their derivatives, are integral valued at 0 and $\alpha$. They are

1. rational $\alpha, f(z) \in Q[x]$,

2. $\alpha$, the logarithm of a rational number, $f(z) \in Q\left[e^{z}, e^{-z}\right]$,

3. $\cosh \sqrt{ } d \alpha$ is rational of the form $\left(1+d r^{2}\right)^{1 / 2}, r$ rational, $d$ squarefree,

$$
f(z) \in Q[\cosh \sqrt{ } d z, \sinh \sqrt{ } d z / \sqrt{ } d] .
$$

\section{BIBLIOGRAPHY}

1. E. G. Straus, On entire functions with algebraic derivatives at certain algebraic points, Ann. of Math. 52 (1950), 188-198.

2. G. Pólya, Uber ganzwertige ganze Funktionen, Rend. Circ. Mat. Palermo 40 (1915), 1-16.

3. G. H. Hardy, On a theorem of Mr. G. Pólya, Proc. Cambridge Philos. Soc. 19 (1916-1919), 60-63.

4. Th. Schneider, Ein Satz über ganzwertige Funktionen als Prinzip für Transzendenzbeweise, Math. Ann. 121 (1949), 131-140.

5. G. Pólya, Uber die kleinsten ganzen Funktionen deren sämtliche Derivierte im Punkte $z=0$ ganzzahlig sind, Tôhoku Math. J. 19 (1921), 65.

6. S. Kakeya, Notes on the maximum modulus of a function, Tôhoku Math. J. 10 (1916), 68.

7. E. C. Titchmarsh, The theory of functions, Oxford Univ. Press, New York, 1939.

8. Th. Schneider, Einführung in die transzendenten Zahlen, Springer-Verlag, Berlin, 1957.

9. W. J. Le Veque, Topics in number theory, Addison-Wesley, Reading, Mass., 1956.

10. R. C. Buck, Integral valued entire functions, Duke Math. J. 15 (1948), 879-891.

UNIVERSITY OF BRITISH COLUMBIA; VANCOUVER, CANADA 